\title{
Hematocrit and the incidence of stroke: a prospective, population-based cohort study
}

This article was published in the following Dove Press journal:

Therapeutics and Clinical Risk Management

\author{
Renling Yang, ${ }^{1,2}$ Anxin \\ Wang, ${ }^{3-6}$ Li Ma, ${ }^{2}$ Zhaoping \\ $\mathrm{Su},{ }^{7}$ Shuohua Chen, ${ }^{8}$ Yilong \\ Wang, ${ }^{3-6}$ Shouling Wu, ${ }^{8}$ \\ Chen Wang ${ }^{1,2}$ \\ 'Department of General Practice, \\ School of General Practice and \\ Continuing Education, Capital Medical \\ University, Beijing, China; ${ }^{2}$ Department \\ of General Practice, Beijing Tiantan \\ Hospital, Capital Medical University, \\ Beijing, China; ${ }^{3}$ Department of \\ Neurology, Beijing Tiantan Hospital, \\ Capital Medical University, Beijing, \\ China; ${ }^{4}$ China National Clinical \\ Research Center for Neurological \\ Diseases, Beijing, China; ${ }^{5}$ Center of \\ Stroke, Beijing Institute for Brain \\ Disorders, Beijing, China; ${ }^{6}$ Beijing Key \\ Laboratory of Translational Medicine \\ for Cerebrovascular Disease, Beijing, \\ China; ${ }^{7}$ Department of Epidemiology \\ and Health Statistics, Academy of \\ Public Health and Management, \\ Weifang Medical University, Weifang, \\ China; ${ }^{8}$ Department of Cardiology, \\ Kailuan Hospital, North China \\ University of Science and Technology, \\ Tangshan, China
}

Correspondence: Chen Wang Department of General Practice, Beijing Tiantan Hospital, Capital Medical University, 6 TiantanXili, Beijing I00050, China

Tel +86 1067098009

Fax +86 I0 67028089

Email wangchen-tr2002@I63.com

Shouling Wu

Department of Cardiology, Kailuan Hospital, North China University of Science and Technology, 57 Xinhua East Road, Tangshan 63000, China

$\mathrm{Tel} / \mathrm{fax}+863153025655$

Email drwusl@।63.com
Background and purpose: Whether higher hematocrit levels could increase the incidence of stroke has always been full of controversy. This study aimed to explore the association between hematocrit and the incidence of stroke in the Chinese population.

Subjects and methods: The Kailuan study is a prospective longitudinal cohort study on risk factors and events of chronic diseases. Using a multivariable Cox proportional hazards analysis, we examined the association between baseline hematocrit values and the incidence of stroke in the Kailuan cohort $(93,299$ participants).

Results: A total of 3,624 participants developed stroke during the 9-year follow-up period. In Cox regression models adjusted for demographic information and for clinical variables, there was a significant association between baseline hematocrit levels and the incidence of stroke. The highest hematocrit quartile (quartile 5: men, hematocrit $>48.6 \%$; women, hematocrit $>43.2 \%$ ) was associated with a higher incidence of stroke (HR 1.17, 95\% CI 1.04-1.31, $P$ for trend $=0.0016$ ) compared with the lowest hematocrit quartile (quartile 1 : men, hematocrit $<41.5 \%$; women, hematocrit $<36.6 \%$ ). In the analysis of ischemic stroke, intracerebral hemorrhage separately, similar association was observed in ischemic stroke, but there were no statistical differences in intracerebral hemorrhage.

Conclusion: Higher hematocrit levels are associated with a higher incidence of stroke in the Chinese population.

Keywords: hematocrit, stroke, cohort

\section{Plain language summary}

Studies have shown that hematocrit might play an important role in blood viscosity and may be an independent risk factor for stroke. Several observational studies have examined the association between hematocrit levels and the incidence of stroke; however, the findings have been inconsistent. The current findings demonstrate that higher hematocrit values were associated with a higher incidence of stroke in the Chinese population. The study also highlights how the association between hematocrit and stroke differs by gender and stroke subtype.

\section{Introduction}

Hematocrit is the ratio of the volume occupied by red blood cells to the volume of the whole blood. Hemoglobin concentration refers to the amount of hemoglobin per unit volume of blood. Hematocrit and hemoglobin play important roles in blood viscosity. ${ }^{1,2}$ Several observational studies have examined the association between hematocrit or hemoglobin levels and the incidence of stroke; however, the findings have been inconsistent. ${ }^{3-9}$ The association has been investigated mainly in the Western population. Higher hematocrit levels were reported to be a risk factor for stroke in 
these studies, such as the Framingham Study, ${ }^{4}$ the British Heart Study ${ }^{5}$ and the Edinburgh Artery Study, ${ }^{6}$ while the results of studies on Asian population are different. ${ }^{3,8}$ In addition, some studies have reported that the association differs by gender. For example, the REGARDS study ${ }^{9}$ discovered a relationship between hemoglobin and stroke only among women, whereas such association was present among men in the Korean Heart Study. ${ }^{7}$ Furthermore, the stroke is classified into three subtypes: ischemic stroke, intracerebral hemorrhage and subarachnoid hemorrhage which have different etiologies; however, few studies investigate the association between hematocrit and stroke subtypes separately.

The purpose of the current study was to investigate the association of hematocrit levels with the development of stroke and the influence of gender on this association in a Chinese population from the Kailuan cohort established in 2006 .

\section{Subjects and methods Study design and population}

The Kailuan study is a prospective, population-based cohort study aimed at exploring the risk factors for chronic diseases (such as stroke and myocardial infarction). ${ }^{10}$ The site of this study is the Kailuan community, a functional community of approximately 150,000 residents in Tangshan, China. The rationale, methods and examination details of the study have been previously published. ${ }^{10}$ Overall, 101,510 individuals aged 18 years or older were enrolled between June 2006 and October 2007 in the Kailuan cohort. After exclusion of 3,669 subjects who had a history of stroke or myocardial infarction and 4,542 for whom hematocrit data were lacking, the remaining 93,299 subjects (73,926 men and 19,373 women) were enrolled in the current study (Figure 1). Our study was approved by the ethics committee of Kailuan General Hospital. All participants signed informed consent forms and completed questionnaire interviews, physical examinations and laboratory tests.

\section{Measurement of hematocrit}

The element of interest was hematocrit levels measured in baseline blood samples using a Hitachi 747 automated hematology analyzer (Hitachi Ltd., Tokyo, Japan) at the central laboratory of the Kailuan General Hospital. Hematocrit is affected by circulating blood volume; hence, the blood samples were collected in the morning after an overnight fast. This method is a commonly used measurement of hematocrit in China. The normal range of hematocrit in China is $40 \%-50 \%$ for men and $37 \%-48 \%$ for women.

\section{Assessment of potential covariates}

The demographic information (age, sex), lifestyle characteristics (smoking status, drinking status) and medical history (hypertension, diabetes, dyslipidemia and atrial fibrillation) were obtained using questionnaires that were

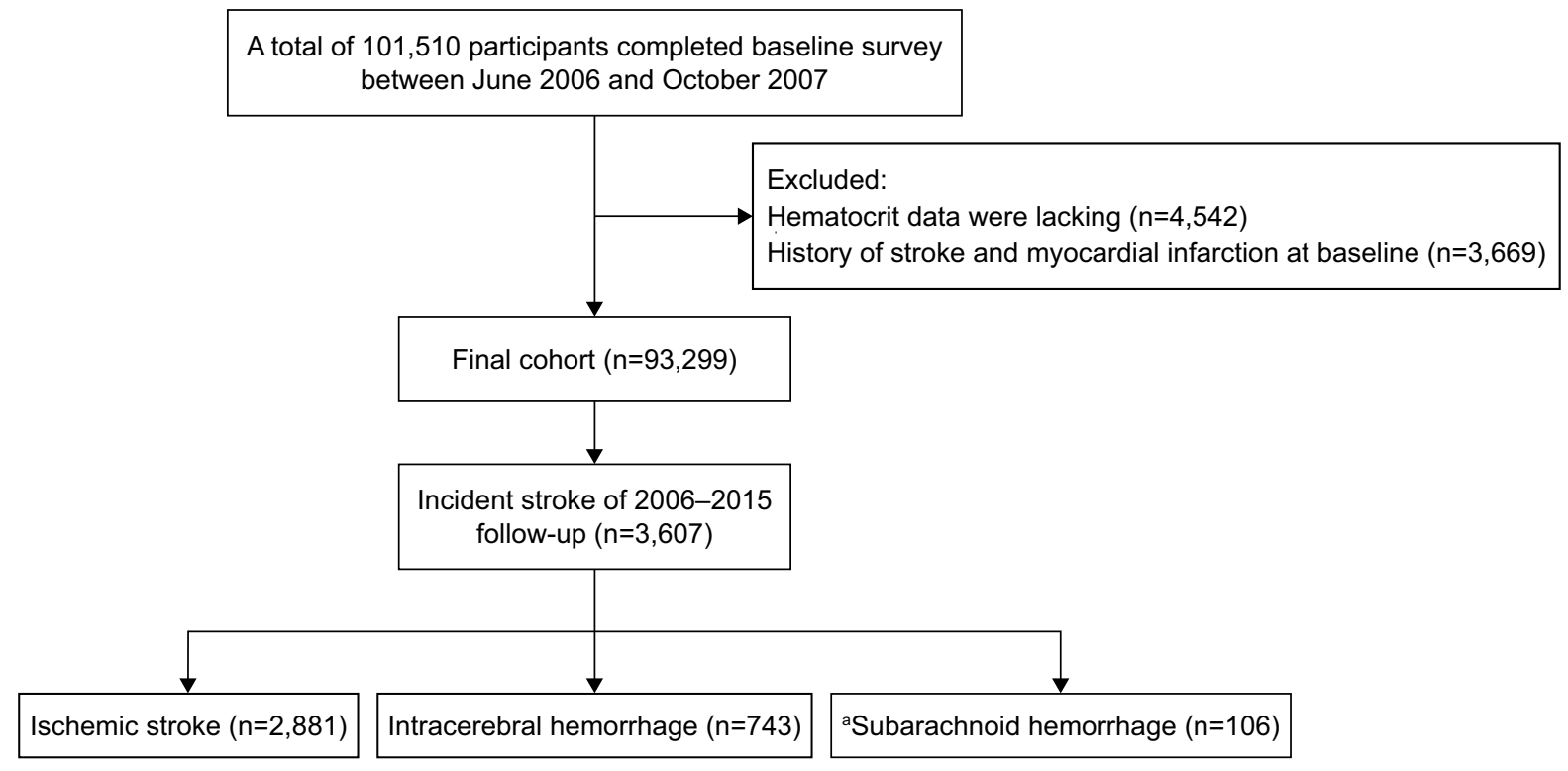

Figure I Study flow diagram depicting exclusion criteria and outcomes. Note: aSubarachnoid hemorrhage was excluded from analyses. 
conducted by professional doctors from the hospitals. Blood pressure was determined as an average of threeseated measurements using a mercury sphygmomanometer. Height and weight were measured without shoes, and body mass index $\left(\mathrm{kg} / \mathrm{m}^{2}\right)$ was calculated as weight divided by the square of height.

Blood samples were collected in the morning after an overnight fast and measured using a Hitachi 747 automated hematology analyzer as mentioned earlier. Fasting plasma glucose (FPG) was tested using the hexokinase/glucose-6phosphate dehydrogenase method. Levels of triglycerides (TGs), total cholesterol (TC), high-density lipoprotein (HDL) and low-density lipoprotein (LDL) were all measured enzymatically. Estimated glomerular filtration rate (eGFR) was calculated using the Chronic Kidney Disease Epidemiology Collaboration equation.

As reported previously, ${ }^{10-12}$ hypertension was ascertained from self-reported history of hypertension, any treatment of hypertension or $\mathrm{SBP} \geq 140 \mathrm{mmHg}$ or $\mathrm{DBP} \geq 90 \mathrm{mmHg}$. Diabetes was ascertained from self-reported history of diabetes, any treatment of diabetes or fasting glucose level $\geq 7.0 \mathrm{mmol} / \mathrm{L}$. Dyslipidemia was determined by selfreported history of dyslipidemia, any treatment of dyslipidemia or serum $\mathrm{TG} \geq 1.69 \mathrm{mmol} / \mathrm{L}, \mathrm{LDL} \geq 3.62 \mathrm{mmol} / \mathrm{L}$ or $\mathrm{HDL} \leq 1.04 \mathrm{mmol} / \mathrm{L}$. Atrial fibrillation was ascertained from detection via electrocardiogram, self-reported history of atrial fibrillation or any treatment of atrial fibrillation.

\section{Follow-up and outcome assessment}

The participants were followed up by trained physicians or nurses until December 31, 2015. The outcomes of interest were mainly collected through face-to-face interviews at health examinations every 2 years. As for participants who did not attend the routine health examination (Table S1), the outcome information was obtained by checking medical insurance records and death certificates from provincial vital statistics offices. Then, the outcome information was validated by reviewing the medical records from the 11 local hospitals.

The primary outcome was the first occurrence of stroke, which was diagnosed based on a combination of symptoms of neurological deficits and brain computed tomography or magnetic resonance imaging according to the WHO criteria. ${ }^{13}$ All stroke events were established by the Arbitration Committee for Clinical Outcomes and the Data Safety Monitoring Board. The outcomes were classified into three subtypes: ischemic stroke, intracerebral hemorrhage and subarachnoid hemorrhage. Because of the low incidence of subarachnoid hemorrhage, we excluded patients with subarachnoid hemorrhage from the following analyses.

\section{Statistical analyses}

Given the differences in the distribution of hematocrit values by sex, the subjects were classified into five groups according to sex-specific quartiles of hematocrit levels. Male and female participants were divided into five quintiles separately according to hematocrit. The five quintiles (Q1-Q5) of the total population were composed of the corresponding parts of male and female participants (men: Q1, <41.5\%; Q2, 41.6\%-44.0\%; Q3, 44.0\%-45.9\%; Q4, 46.0\%-48.5\%; and Q5, >48.6\%; women: Q1, <36.6\%; Q2, 36.7\%-38.8\%; Q3, 38.9\%-40.7\%; Q4, 40.7\%-43.1\%; and $\mathrm{Q} 5,>43.2 \%$ ). Descriptive statistics were used to present the participant characteristics across the quartiles of baseline hematocrit values. The continuous variables are described by mean and SD, and the categorical variables are described by percentages. A test for linear trend was performed to determine the trends of characteristics within the hematocrit quartiles.

Cox proportional hazards analysis was used to examine the associations of baseline hematocrit levels with the incidences of stroke, ischemic stroke and hemorrhagic stroke by treating the lowest quartile as the reference in sequential models. The choice of adjusted factors was mainly based on two considerations which on the one hand was from the statistically significant factors of test for linear trend and on the other hand was from the factors reported in the literature that have an impact on stroke. Model 1 was adjusted for age and sex. Model 2 was adjusted for variables in model 1 plus body mass index, smoking, drinking, dyslipidemia, diabetes mellitus and hypertension. Model 3 was adjusted for variables in model 2 plus atrial fibrillation, SBP, FPG, TC, LDL and eGFR. In addition, a test for linear trend was performed for each model. Further, we conducted a sensitivity analysis to test the robustness of our findings. Hypertension is an important risk factor for stroke, and some people developed hypertension during the follow-up period. So, we repeated our analysis after excluding these individuals. Finally, because previous studies showed that the association of hematocrit with stroke was modified by sex, ${ }^{3,7,9}$ we tested whether there was an interaction of sex and baseline hematocrit levels (hematocrit $\times$ sex). Two-sided $P$-values are reported for all analyses. A value of $P<0.05$ was considered to be statistically significant. All statistical analyses were performed by SAS software version 9.2 (SAS Institute Inc., Cary, NC, USA). 


\section{Results}

In the current study, men composed $79.24 \%$ of the entire population. The mean values of hematocrit were $45.1 \%$ for men and $40.0 \%$ for women. During a mean follow-up of $8.85 \pm 1.03$ years, 2,881 participants had ischemic stroke and 743 had hemorrhagic stroke (Figure 1). The baseline characteristics of the study population according to the quartiles of hematocrit values are summarized in Table 1. When compared with the participants with lower hematocrit levels, the individuals with higher hematocrit levels were more likely to be younger, had a lower eGFR level, had higher prevalences of smoking and drinking, hypertension, diabetes mellitus and dyslipidemia and had higher SBP, DBP, BMI, TG, LDL and TC levels.

Table 2 summarizes the HRs with 95\% CIs for stroke and its subtypes according to the hematocrit quartiles and $P$-values from the models. There was no significant association between hematocrit and stroke in the unadjusted model. However, in the multivariate-adjusted analyses, the hematocrit levels were positively correlated with the incidence of total stroke $(P<0.01$ for all). Compared with the first hematocrit quartile as a reference, the highest quartile had a significantly higher age- and sex-adjusted incidence of stroke in model 1 (HR 1.50, 95\% CI 1.34-1.67). After adjusting for the demographic factors and laboratory indices in model 2 and model 3, the association between hematocrit and stroke incidence was attenuated but remained significant. The adjusted HR (95\% CI) for stroke in model 2 was 1.25 (1.12-1.39) and 1.17 (1.04-1.31) in model 3. In the sensitivity analysis, the associations remained significant after excluding individuals who developed hypertension during the follow-up (HR 1.17, 95\% CI 1.04-1.31). In terms of the subtypes of stroke, the incidence of ischemic stroke was increased significantly in the highest quartile in the three models. However, no significant association was observed with the incidence of hemorrhagic stroke.

There were no statistical interactions between the hematocrit and sex. However, sex-specific analyses were carried out for the sake of interest, because the normal ranges of hematocrit are generally higher in men than in women. As shown in Figure 2, the relationship between hematocrit and stroke exhibits gender differences. As in the total population, the hematocrit levels were also linearly correlated with the incidence of stroke in men (model 3: HR 1.20, 95\% CI $1.07-1.35, P$ for trend $<0.001)$, but there was no significant association between hematocrit and stroke in women.

\section{Discussion}

Using the data from a long follow-up of the Kailuan community, we found that the incidence of stroke was significantly increased in subjects with higher hematocrit levels and this association was mainly found among ischemic stroke subtype

Table I Baseline characteristics of study participants by quartiles of hematocrit

\begin{tabular}{|c|c|c|c|c|c|c|}
\hline & Hematocrit QI & Hematocrit Q2 & Hematocrit Q3 & Hematocrit Q4 & Hematocrit Q5 & $P$ for trend \\
\hline $\mathrm{N}$ & 18,447 & 18,503 & $|8,6| \mid$ & 19,190 & 18,548 & \\
\hline Age, years & $55.21 \pm \mid 3.21$ & $52.53 \pm 12.33$ & $51.16 \pm 12.08$ & $50.20 \pm 12.09$ & $48.83 \pm 12.09$ & $<0.001$ \\
\hline Men, n (\%) & $|4,6| 3(79.22)$ & $14,542(78.56)$ & $14,7 \mid 6$ (79.07) & $15,426(80.39)$ & 14,629 (78.87) & 0.23 \\
\hline Current smoking, n (\%) & $4,918(26.22)$ & $5,723(30.93)$ & $6,082(32.68)$ & $6,742(35.13)$ & $7,034(37.92)$ & $<0.001$ \\
\hline Current drinking, n (\%) & $5,346(28.98)$ & $6,137(33.17)$ & $6,662(35.80)$ & $7,242(37.74)$ & $7,846(42.43)$ & $<0.001$ \\
\hline Body mass index, $\mathrm{kg} / \mathrm{m}^{2}$ & $24.28 \pm 3.54$ & $24.76 \pm 3.45$ & $25.08 \pm 3.44$ & $25.34 \pm 3.42$ & $25.66 \pm 3.47$ & $<0.001$ \\
\hline Hypertension, n (\%) & $7,552(40.94)$ & $7,846(42.40)$ & $8,090(43.47)$ & $8,739(45.54)$ & 8,307 (44.79) & $<0.001$ \\
\hline Diabetes mellitus, n (\%) & $\mathrm{I}, 425(7.72)$ & I,593 (8.6I) & $1,569(8.43)$ & I,892 (9.86) & I,940 (10.46) & $<0.001$ \\
\hline Dyslipidemia, n (\%) & $5,64 \mid(30.58)$ & 6,178 (33.39) & $6,512(34.99)$ & $6,956(36.25)$ & $6,890(37.15)$ & $<0.001$ \\
\hline Atrial fibrillation, $\mathrm{n}(\%)$ & $89(0.48)$ & $68(0.37)$ & $69(0.37)$ & $82(0.43)$ & $90(0.49)$ & 0.66 \\
\hline $\mathrm{SBP}, \mathrm{mmHg}$ & $129.98 \pm 21.12$ & $|30.45 \pm 20.7|$ & $|30.77 \pm 20.5|$ & $|3| .56 \pm 20.80$ & $131.19 \pm 21.13$ & $<0.001$ \\
\hline $\mathrm{DBP}, \mathrm{mmHg}$ & $81.64 \pm \mid 1.51$ & $83.05 \pm 11.56$ & $83.64 \pm 11.53$ & $84.43 \pm 11.82$ & $84.53 \pm 12.12$ & $<0.001$ \\
\hline $\mathrm{FPG}, \mathrm{mmol} / \mathrm{L}$ & $5.32 \pm 1.58$ & $5.42 \pm 1.63$ & $5.44 \pm 1.57$ & $5.55 \pm 1.78$ & $5.55 \pm 1.80$ & $<0.001$ \\
\hline $\mathrm{TC}, \mathrm{mmol} / \mathrm{L}$ & $4.77 \pm 1.14$ & $4.87 \pm 1.14$ & $4.94 \pm 1.15$ & $5.03 \pm 1.14$ & $5.08 \pm 1.15$ & $<0.001$ \\
\hline $\mathrm{LDL}, \mathrm{mmol} / \mathrm{L}$ & $2.15 \pm 1.03$ & $2.29 \pm 0.97$ & $2.38 \pm 0.92$ & $2.44 \pm 0.86$ & $2.42 \pm 0.78$ & $<0.001$ \\
\hline $\mathrm{HDL}, \mathrm{mmol} / \mathrm{L}$ & $1.57 \pm 0.43$ & $1.54 \pm 0.4 \mathrm{I}$ & $\mathrm{I} .54 \pm 0.4 \mathrm{I}$ & $1.55 \pm 0.39$ & $1.57 \pm 0.39$ & 0.58 \\
\hline TGs, mmol/L & $1.54 \pm 1.35$ & $1.63 \pm 1.40$ & $1.69 \pm 1.39$ & $1.75 \pm 1.39$ & $1.76 \pm 1.37$ & $<0.001$ \\
\hline EGFR, $\mathrm{mL} / \mathrm{min}$ per $1.73 \mathrm{~m}^{2}$ & $82.87 \pm 29.74$ & $83.18 \pm 25.22$ & $82.43 \pm 23.25$ & $81.39 \pm 25.92$ & $79.37 \pm 24.30$ & $<0.001$ \\
\hline
\end{tabular}

Notes: Hematocrit levels were classified into sex-specific quartiles (men: QI, <41.5\%; Q2, 41.6\%-44.0\%; Q3, 44.0\%-45.9\%; Q4, 46.0\%-48.5\%; and Q5, >48.6\%; women: QI, <36.6\%; Q2, 36.7\%-38.8\%; Q3, 38.9\%-40.7\%; Q4, 40.7\%-43.1\%; and Q5, >43.2\%).

Abbreviations: EGFR, EGF receptor; FPG, fasting plasma glucose; HDL, high-density lipoprotein; LDL, low-density lipoprotein; TC, total cholesterol; TG, triglyceride; Q, quintile. 
Table 2 HRs $(95 \% \mathrm{Cl})$ of incident stroke according to quartiles of baseline hematocrit

\begin{tabular}{|c|c|c|c|c|c|c|}
\hline & QI & Q2 & Q3 & Q4 & Q5 & $P$ for trend \\
\hline Stroke, n (\%) & $747(4.05)$ & $709(3.83)$ & $694(3.73)$ & $75 I(3.9 I)$ & $706(3.8 I)$ & \\
\hline Crude HR (95\% Cl) & 1.0 & $0.99(0.89-1.10)$ & $\mathrm{I} .00(0.90-\mathrm{I} . \mathrm{II})$ & $1.08(0.97-1.20)$ & $1.07(0.96-1.19)$ & 0.08 \\
\hline Model I HR $(95 \%$ Cl) & 1.0 & $1.16(1.04-1.28)$ & $1.25(1.12-1.39)$ & $1.40(1.26-1.55)$ & $1.50(1.34-1.67)$ & $<0.001$ \\
\hline Model $2 \mathrm{HR}^{\mathrm{b}}(95 \% \mathrm{Cl})$ & 1.0 & $1.08(0.97-1.20)$ & $1.13(1.01-1.25)$ & $1.21(1.09-1.35)$ & $1.25(1.12-1.39)$ & $<0.001$ \\
\hline Model $3 \mathrm{HR}^{\mathrm{c}}(95 \% \mathrm{Cl})$ & 1.0 & $1.05(0.95-1.17)$ & $1.09(0.98-1.21)$ & $1.16(1.04-1.29)$ & $1.17(1.04-1.31)$ & 0.0016 \\
\hline Ischemic stroke, n (\%) & $582(3.15)$ & $563(3.04)$ & $557(2.99)$ & $625(3.26)$ & 554 (2.99) & \\
\hline Crude HR $(95 \% \mathrm{Cl})$ & 1.0 & $1.01(0.90-1.32)$ & $1.03(0.92-1.16)$ & $1.16(1.03-1.30)$ & $1.08(0.95-1.22)$ & 0.04 \\
\hline Model I HRa $(95 \% \mathrm{Cl})$ & 1.0 & $1.19(1.06-1.34)$ & $1.31(1.16-1.47)$ & $1.53(1.36-1.72)$ & $1.54(1.36-1.75)$ & $<0.001$ \\
\hline Model $2 \mathrm{HR}^{\mathrm{b}}(95 \% \mathrm{Cl})$ & 1.0 & I.II (0.99-I.25) & $1.18(1.05-1.33)$ & $1.32(1.17-1.48)$ & $1.28(1.13-1.45)$ & $<0.001$ \\
\hline Model $3 \mathrm{HR}^{\mathrm{c}}(95 \% \mathrm{Cl})$ & 1.0 & $1.09(0.96-1.22)$ & $1.14(1.01-1.28)$ & $1.25(|| I-.|.4|)$ & $1.19(1.05-1.35)$ & $<0.001$ \\
\hline Intracerebral hemorrhage, $n$ (\%) & $170(0.92)$ & $143(0.77)$ & $143(0.77)$ & $140(0.73)$ & 147 (0.79) & \\
\hline Crude HR $(95 \% \mathrm{Cl})$ & 1.0 & $0.88(0.70-1.10)$ & $0.90(0.72-1.12)$ & $0.87(0.69-1.10)$ & $1.00(0.79-1.26)$ & 0.88 \\
\hline Model I HR $(95 \% \mathrm{Cl})$ & 1.0 & $0.99(0.79-1.24)$ & $1.07(0.85-1.34)$ & $1.07(0.85-1.35)$ & $1.30(1.03-1.64)$ & 0.04 \\
\hline Model $2 \mathrm{HR}^{\mathrm{b}}(95 \% \mathrm{Cl})$ & 1.0 & $0.93(0.75-1.17)$ & $0.97(0.77-1.22)$ & $0.94(0.75-1.19)$ & I.II (0.87-I.4I) & 0.49 \\
\hline Model $3 \mathrm{HR}^{\mathrm{c}}(95 \% \mathrm{Cl})$ & 1.0 & $0.93(0.74-1.16)$ & $0.96(0.76-1.21)$ & $0.93(0.73-1.17)$ & $1.08(0.85-1.38)$ & 0.61 \\
\hline Sensitivity analysis $^{d}$ & 1.0 & $1.05(0.94-1.16)$ & $1.10(0.99-1.23)$ & $1.17(1.05-1.30)$ & $1.17(1.04-1.31)$ & 0.0011 \\
\hline
\end{tabular}

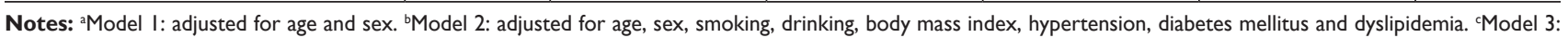
adjusted for age, sex, smoking, drinking, body mass index, hypertension, diabetes mellitus, dyslipidemia, atrial fibrillation, SBP, FPG, TC, LDL and estimated glomerular and proteinuria. ${ }^{\mathrm{A}}$ Adjusted for model 3 and further excluded individuals who developed hypertension during the follow-up.

Abbreviations: FPG, fasting plasma glucose; LDL, low-density lipoprotein; TC, total cholesterol; Q, quintile.

and male participants. These findings emphasize the clinical importance of high hematocrit level as a risk factor for stroke in the Chinese population.

Previous studies have highlighted the association between hematocrit and stroke incidence. Gagnon et $\mathrm{al}^{4}$ examined this association in the Framingham cohort. Their results revealed that hematocrit was an important risk factor for some cardiovascular events, but the hematocrit produced different effect on stroke events of different age and sex groups. Later, two follow-up studies, one from the British Heart Study ${ }^{5}$ and one

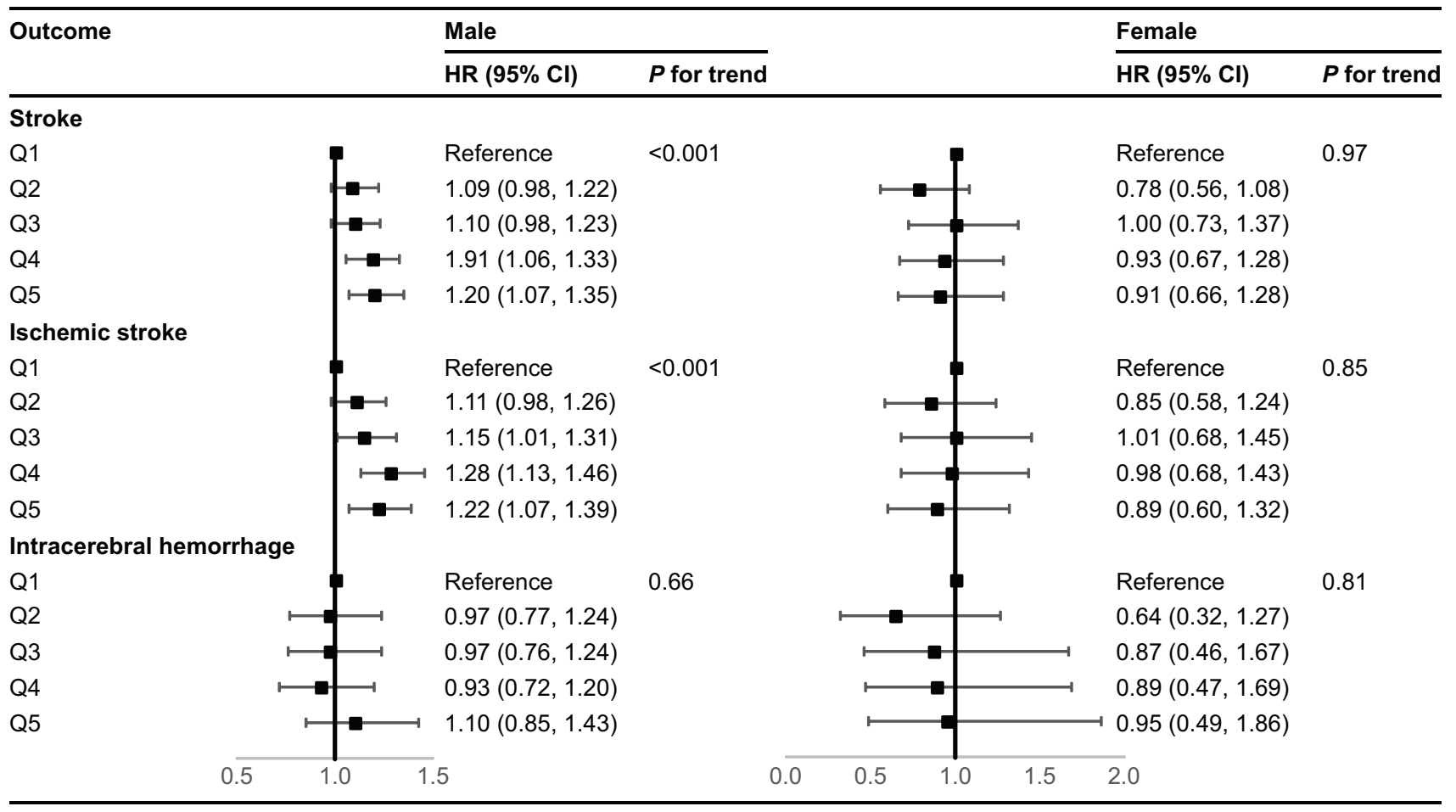

Figure 2 HRs $(95 \% \mathrm{Cl})$ of incident stroke according to sex-specific quartiles of baseline hematocrit. 
from the Edinburgh Artery Study, ${ }^{6}$ reported that high hematocrit levels were associated with a higher incidence of stroke, which is similar to our finding. In addition, some studies also found that a higher level of hematocrit was an independent risk factor for other vascular diseases, such as ischemic heart disease $^{14}$ and venous thromboembolism, ${ }^{15,16}$ which echo the association between hematocrit and stroke. Therefore, our study adds to this literature by showing the linear association between hematocrit and stroke incidence. There were also some studies that presented different views. For example, a Japanese study from the Hisayama cohort by Kiyohara et $\mathrm{al}^{3}$ found that lower hematocrit levels will increase the incidence of ischemic stroke. However, fewer events occurred in this study, so the level of evidence is weak. In the same cohort, another study published in 2015 by Gotoh et $\mathrm{al}^{8}$ showed that higher hematocrit levels increased the incidence of ischemic stroke, which is in agreement with our study. However, lower hematocrit levels were considered to be a risk factor for hemorrhagic stroke in Gotoh et al's study, while in our study, there was no significant relationship between lower hematocrit and hemorrhagic stroke. This difference could be explained partially by the study population, as the mean hematocrit values and the incidence of hemorrhagic stroke in our study were lower than those in the Japanese study.

Several pathophysiological mechanisms could potentially explain the association of high hematocrit levels with an increased ischemic stroke incidence. Elevated hematocrit levels lead to increased blood viscosity and peripheral resistance, thereby reducing cerebral circulation. ${ }^{1,2,17}$ In addition, high hematocrit levels increase peripheral platelet activation by releasing erythrocyte-derived ADP. ${ }^{18,19}$ Increased hematocrit levels may also contribute to developing ischemic stroke by other mechanisms, such as oxidative stress, lipid peroxidation ${ }^{20}$ and thrombin generation. ${ }^{21,22}$ The mechanism why high hematocrit levels increased intracerebral hemorrhage incidence is unclear. Some studies have reported that high hematocrit levels are associated with the onset of hypertension. In addition, hypertension is a significant risk factor for hemorrhagic stroke, so high hematocrit levels may affect intracerebral hemorrhage by blood pressure.

The differential associations of hematocrit and stroke incidence with sexes could be explained by the different hematocrit distribution between men and women. The mean hematocrit level of the fifth quartile in men was higher than that in women (46.3\% for women vs $51.6 \%$ for men, $P<0.001)$. In addition, age-specific stroke rates are higher in men than women. Adult males have a higher stroke incidence than age-matched females. ${ }^{23,24}$ Because of declining estrogen levels, the risk of stroke in women increases after menopause, but women also have lower stroke rates than men. Therefore, the hematocrit level of most women might not be high enough to contribute to stroke.

The strengths of our study include the prospective study design, a large study sample size in the Chinese population, a long follow-up period and an accurate method for stroke diagnosis through reviewing medical records. However, some limitations of our study should be mentioned. First, our findings were based on baseline hematocrit measurements. However, the hematocrit levels may change during the follow-up period, so we cannot determine whether any changes in hematocrit levels were associated with stroke incidence. Second, although we attempted to account for possible confounding factors, residual confounders might still exist in our analysis such as anticoagulants and antiplatelets. However, we could not collect this information because Kailuan database did not include them. Next, most participants of the Kailuan study are male coal miners, so the study population might not represent the general Chinese population. In addition, because our findings are based on an observational study design, we cannot make definite causal inferences between high hematocrit levels and stoke incidence.

\section{Conclusion}

Our findings suggest that higher hematocrit levels are associated with a higher incidence of stroke in the Chinese population and the influence of hematocrit is mainly in ischemic stroke. The hematocrit levels should be taken into consideration when assessing the incidence of stroke.

\section{Acknowledgments}

We appreciate the survey teams in Kailuan General Hospital and management teams in Beijing Tiantan Hospital. We also thank Professor Yongjun Wang for his help in the revision of the paper. Funding for this study was provided by the Prevention and Control of Major Chronic Noncommunicable Diseases Program of China (2016YFC0901001 and 2017YFC1310900), Beijing Municipal Science and Technology Commission (D151100002015003) and Beijing Municipal Administration of Hospitals' Mission Plan (SML20150502).

\section{Disclosure}

The authors report no conflicts of interest in this work.

\section{References}

1. Wood JH, Kee DB. Hemorheology of the cerebral circulation in stroke. Stroke. 1985;16(5):765-772. 
2. de Simone G, Devereux RB, Chien S, Alderman MH, Atlas SA, Laragh JH. Relation of blood viscosity to demographic and physiologic variables and to cardiovascular risk factors in apparently normal adults. Circulation. 1990;81(1):107-117.

3. Kiyohara Y, Ueda K, Hasuo Y, et al. Hematocrit as a risk factor of cerebral infarction: long-term prospective population survey in a Japanese rural community. Stroke. 1986;17(4):687-692.

4. Gagnon DR, Zhang TJ, Brand FN, Kannel WB. Hematocrit and the risk of cardiovascular disease-The Framingham study: A 34-year follow-up. Am Heart J. 1994;127(3):674-682.

5. Wannamethee G, Perry IJ, Shaper AG, Haematocrit SAG. Haematocrit, hypertension and risk of stroke. J Intern Med. 1994;235(2):163-168.

6. Lowe GD, Lee AJ, Rumley A, Price JF, Fowkes FG. Blood viscosity and risk of cardiovascular events: the Edinburgh Artery Study. $\mathrm{Br} J$ Haematol. 1997;96(1):168-173.

7. Kim MY, Jee SH, Yun JE, Baek SJ, Lee DC. Hemoglobin concentration and risk of cardiovascular disease in Korean men and women - the Korean heart study. J Korean Med Sci. 2013;28(9):1316-1317.

8. Gotoh S, Hata J, Ninomiya T, et al. Hematocrit and the risk of cardiovascular disease in a Japanese community: The Hisayama Study. Atherosclerosis. 2015;242(1):199-204.

9. Panwar B, Judd SE, Warnock DG, et al. Hemoglobin concentration and risk of incident stroke in community-living adults. Stroke. 2016;47(8):2017-2024.

10. Wang A, Wu J, Zhou Y, et al. Measures of adiposity and risk of stroke in China: a result from the Kailuan study. PLoS One. 2013;8(4): e61665-e61668.

11. Wang A, Liu X, Su Z, et al. Two-year changes in proteinuria and risk for myocardial infarction in patients with hypertension: a prospective cohort study. J Hypertens. 2017;35(11):2295-2302.

12. Wang A, Sun Y, Liu X, et al. Changes in proteinuria and the risk of myocardial infarction in people with diabetes or pre-diabetes: a prospective cohort study. Cardiovasc Diabetol. 2017;16(1):104.

13. Listed N. Stroke - 1989. Recommendations on stroke prevention, diagnosis, and therapy. Report of the WHO Task Force on Stroke and other Cerebrovascular Disorders. Stroke. 1989;20(10):1407-1431.
14. Wannamethee G, Shaper AG, Whincup PH. Ischaemic heart disease: association with haematocrit in the British Regional Heart Study. J Epidemiol Community Health. 1994;48(2):112-118.

15. Braekkan SK, Mathiesen EB, Njølstad I, Wilsgaard T, Hansen JB. Hematocrit and risk of venous thromboembolism in a general population. The Tromso study. Haematologica. 2010;95(2):270-275.

16. Eischer L, Tscholl V, Heinze G, Traby L, Kyrle PA, Eichinger S. Hematocrit and the risk of recurrent venous thrombosis: a prospective cohort study. PLoS One. 2012;7(6):e38705.

17. Burch GE, Depasquale NP, Hematocrit DNP. Hematocrit, viscosity and coronary blood flow. Dis Chest. 1965;48(3):225-232.

18. Lowe GDO, Forbes CD. Platelet aggregation, haematocrit, and fibrinogen. Lancet. 1985;325(8425):395-396.

19. Praticó D, Pasin M, Barry OP, et al. Iron-dependent human platelet activation and hydroxyl radical formation: involvement of protein kinase C. Circulation. 1999;99(24):3118-3124.

20. Salonen JT, Nyyssönen K, Korpela H, Tuomilehto J, Seppänen R, Salonen R. High stored iron levels are associated with excess risk of myocardial infarction in eastern Finnish men. Circulation. 1992; 86(3):803-811.

21. Peyrou V, Lormeau JC, Hérault JP, Gaich C, Pfliegger AM, Herbert JM. Contribution of erythrocytes to thrombin generation in whole blood. Thromb Haemost. 1999;81(3):400-406.

22. Horne MK 3rd, Cullinane AM, Merryman PK, Hoddeson EK. The effect of red blood cells on thrombin generation. Br J Haematol. 2006; 133(4):403-408.

23. Reeves MJ, Bushnell CD, Howard G, et al. Sex differences in stroke: epidemiology, clinical presentation, medical care, and outcomes. Lancet Neurol. 2008;7(10):915-926.

24. Petrea RE, Beiser AS, Seshadri S, Kelly-Hayes M, Kase CS, Wolf PA. Gender differences in stroke incidence and poststroke disability in the Framingham heart study. Stroke. 2009;40(4):1032-1037. 


\section{Supplementary material}

Table SI Number of participants who did not undergo the routine health examination

\begin{tabular}{l|l|l|l|l}
\hline & 2008-2009 & 20I0-20II & 20I2-20I3 & 20I4-20I5 \\
\hline Absent number $(n)$ & $22,03 I$ & 26,721 & 25,549 & 32,185 \\
Cumulative death $(n)$ & $58 I$ & 1,866 & 3,609 & 5,427 \\
\hline
\end{tabular}

\section{Publish your work in this journal}

Therapeutics and Clinical Risk Management is an international, peerreviewed journal of clinical therapeutics and risk management, focusing on concise rapid reporting of clinical studies in all therapeutic areas, outcomes, safety, and programs for the effective, safe, and sustained use of medicines. This journal is indexed on PubMed Central, CAS,
EMBase, Scopus and the Elsevier Bibliographic databases. The manuscript management system is completely online and includes a very quick and fair peer-review system, which is all easy to use. Visit http://www.dovepress.com/testimonials.php to read real quotes from published authors.

Submit your manuscript here: http://www.dovepress.com/therapeutics-and-clinical-risk-management-journal 